\title{
El impacto de los costes de despido sobre el empleo en España: Una estimación con datos de panel ${ }^{*}$
}

\author{
MARTA MARTÍNEZ MATUTE \\ Departamento de Fundamentos del Análisis Económico e Historia e Instituciones \\ Económicas, UNIVERSIDAD DE VALLADOLID, ESPAÑA. E-mail: martamm@eco.uva.es
} CARLOS PÉREZ DOMÍNGUEZ

Departamento de Fundamentos del Análisis Económico e Historia e Instituciones Económicas, UNIVERSIDAD DE VALLADOLID, ESPAÑA. E-mail: carpe@eco.uva.es

\begin{abstract}
RESUMEN
En este trabajo se analiza el efecto de los costes de despido sobre el nivel de empleo de España. A tal fin, se plantea un modelo de demanda de trabajo de dos periodos con incertidumbre. En dicho modelo, la presencia de futuros costes de despido volverá al empresario más cauto a la hora de contratar nuevos trabajadores en el primer periodo, pero el coste asociado al potencial despido futuro hará que la empresa sea más reticente a disminuir la plantilla en el segundo. Por tanto, el impacto global en el empleo resulta ambiguo, lo que dota de especial importancia al trabajo empírico. Este contraste empírico, en nuestro caso, consiste en plantear un panel dinámico regional para el periodo 2005-2011. El principal resultado obtenido de su estimación econométrica es que, en España, unos costes de despido más altos influyen negativamente en el nivel de empleo en el corto plazo, aunque este efecto se difumina y termina por desaparecer con el paso del tiempo.
\end{abstract}

Palabras clave: Costes de despido, mercado de trabajo español, legislación sobre despido, panel dinámico.

\section{Impact of Firing Costs on the Spanish Employment: A Panel Data Estimation}

\begin{abstract}
This paper examines the effect of firing costs on the Spanish employment. On the basis of a theoretical model with two periods of time and firing costs, a regional dynamic panel data is estimated for the period 2005-2011. According to this model, two opposite effects take place: on the one hand, the presence of firing cost makes the employer more careful to hire new workers in the first period; but, on the other hand, the firing penalty makes the firm more reluctant to reduce employment in period 2 . Then, the global effect of mandatory firing costs remains unclear. The main result obtained in our estimations is that higher firing costs would negatively affect employment in the short run, but in the long run this effect vanishes.

Keywords: Firing Costs, Spanish Labor Market, Firing Legislation, Dynamic Panel.

Clasificación JEL: K31, J32, J63, J65

\footnotetext{
* Los autores agradecen los comentarios recibidos a dos versiones previas de este trabajo que fueron presentadas en el XXV Congreso Internacional de Economía Aplicada ASEPELT 2011 y en las IX Jornadas de Economía Laboral. Nuestro agradecimiento también a los dos evaluadores anónimos de Estudios de Economía Aplicada cuyos comentarios han permitido mejorar la calidad de este articulo. La responsabilidad de las posibles deficiencias es exclusivamente de sus autores.
}

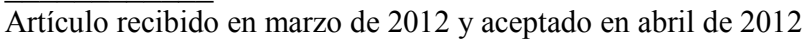

Artículo disponible en versión electrónica en la página www.revista-eea.net, ref. ə-30117 


\section{INTRODUCCIÓN}

Cuando una empresa se enfrenta a una situación económica adversa se le puede plantear la opción de reducir la cantidad de factor trabajo, al igual que puede optar por reducir otro tipo de factores productivos con los que opera, con el objetivo de ahorrar costes a más largo plazo y tener un margen de maniobra mayor que le permita superar sus pérdidas de forma más holgada. Así pues, el despido supone la finalización de un contrato laboral con un empleado por iniciativa del empresario.

La Organización Internacional del Trabajo (OIT) ${ }^{1}$ estipula las directrices básicas para que los países las desarrollen en sus legislaciones propias. En la Unión Europea existen algunas directivas ${ }^{2}$ orientadas a limitar el poder del empresario y a establecer procedimientos legales comunes en relación con el preaviso y la reclamación del trabajador. Podemos seguir la concepción tomada por otros autores (Malo y Toharia, 1999; González y Vacas, 2007) acerca de la existencia de dos modelos de costes de despido claramente diferenciados: el modelo liberal, representado por países anglosajones como Gran Bretaña o Estados Unidos, y el modelo continental europeo, donde tenemos el ejemplo de Francia, Alemania o donde se incluiría España. El modelo liberal es más flexible. La doctrina norteamericana del "empleo a voluntad" (employment-at-will) provoca que la legislación esté prácticamente limitada a algunas normas sobre preaviso o discriminación. Por el contrario, los países europeos, especialmente los mediterráneos, contienen una vasta tradición legislativa de protección al empleo que regulan incluso la obligación de que el empresario busque alternativas al despido o unas altas indemnizaciones y un complejo proceso burocrático que también conlleva unos costes inherentes.

En España existen cuatro tipos de despido principales regulados en el Estatuto de los Trabajadores: el despido disciplinario, el despido colectivo, el despido

\footnotetext{
${ }^{1}$ La Recomendación sobre terminación de la relación de trabajo (núm. 166) de 1982 y el Convenio sobre terminación de la relación de trabajo (núm. 158) de 1982 establecen que debe existir una causa justificada para acometer un despido, con sus consiguientes excepciones (por razones de discriminación, por ejemplo). Éste deberá ir acompañado de un preaviso, de una consulta a los representantes y de una indemnización correspondiente fijada en proporción al tiempo de servicio y al salario recibido y pagada directamente o constituyendo un fondo de cotización del empleador, y además podrá ser recurrido en los tribunales correspondientes siguiendo la ordenación jurídica de cada país. A partir de estos preceptos, cada país está autorizado a ampliar en la medida de sus intereses la legislación correspondiente.

${ }^{2}$ La Directiva 75/129/CEE, de 17 de febrero, del Consejo (completada por la Directiva 92/56/ CEE, de 24 de junio) ofrece una base legal a todos los Estados miembro; la Directiva 98/59/CE, de 20 de julio de 1998, del Consejo, sobre aproximaciones de las legislaciones de los Estados miembros que se refieren a los despidos colectivos; y la Directiva 94/45/CE, de 22 de septiembre, del Consejo, que regula los Comités de Empresa Europeos, donde se incluyen obligaciones de consulta y participación ante situaciones de reducción sustancial de la plantilla.
} 
improcedente o injustificado y el despido por causas objetivas. Su regulación básica está recogida en los artículos 51 al 57 del Estatuto, pero ha sufrido diversas modificaciones en las sucesivas reformas legislativas. Éstas han provocado algunos cambios en cuanto a las indemnizaciones por despido, lo que ha ido configurando el panorama actual.

Han sido numerosos los trabajos teóricos y empíricos que han abordado el estudio de los efectos de los costes de despido sobre el nivel de actividad económica y el empleo. Walter Oi (1962) llevó a cabo uno aportación teórica seminal sobre la materia, concibiendo el trabajo como un factor de producción cuasi-fijo merced a la presencia de costes de despido. En este sentido, y también desde una perspectiva estática, Lazear (1990) plantea cómo los costes de rotación laboral generan ineficiencia en el mercado de trabajo lo que termina revelándose en forma de mermas en el nivel de empleo. De hecho, de sus estimaciones, tomando como referencia una muestra de 22 países, se desprende una relación inversa entre los costes de despido y el nivel de empleo. No obstante, ha sido en el ámbito de los modelos dinámicos de demanda de trabajo donde ha encontrado un sustento teórico más adecuado el estudio del impacto sobre la producción y el empleo de los costes de rotación laboral, en general, y los de despido, en particular. ${ }^{3}$ Entre ellos, los trabajos de Bertola (1990), Bentolila y Bertola (1990), Nickell (1991), Bertola (1992) y Bentolila y Saint-Paul (1992, 1994) inciden en la idea de que los costes de rotación de la mano de obra afectan a la dinámica de la ocupación (ralentizando el ajuste ante las perturbaciones) pero no tienen por qué afectar a los niveles medios de empleo (y desempleo) de largo plazo. Hunt (2000), analizando el impacto de los costes de despido en el empleo alemán, llega también a que dichos costes reducen el output (y el empleo) a corto plazo, mientras que a largo plazo el efecto global termina siendo nulo. Un resultado similar se obtiene, desde el punto de vista teórico, en el trabajo de Eeckhoudt y Treich (2003) mediante un modelo de ajuste de la producción en dos periodos con incertidumbre. ${ }^{4}$ Por último, cabe mencionar el reciente trabajo de Bentolila et al. (2010) en el que los autores señalan "que la brecha en los costes de despido entre trabajadores indefinidos y temporales es el factor singular más importante que induce la dualidad del mercado de trabajo y el exceso de volatilidad del empleo en España."

Nuestro objetivo central en esta investigación es analizar el efecto que los costes de despido tienen sobre el nivel de empleo asalariado de España utilizan-

\footnotetext{
${ }^{3}$ Hamermesh (1995) sintetiza un amplio abanico de investigaciones realizadas hasta ese momento.

${ }^{4}$ Además de la línea comentada, la investigación de Ljungqvist (2002) profundiza en el papel que juegan diferentes modelos de demanda de trabajo en el efecto de los costes de ajuste, concluyendo que los costes de despido incrementan el empleo en modelos de búsqueda mientras que en modelos de loterías los efectos son contrarios, dado que no existe desempleo friccional.
} 
do una muestra reciente de datos. Con este fin, planteamos, en primer lugar, un modelo teórico dinámico de dos periodos con incertidumbre, en la línea de Bentolila y Saint-Paul (1994) y Eeckhoudt y Treich (2003). En dicho modelo, la presencia de futuros costes de despido volverá al empresario más cauto a la hora de contratar nuevos trabajadores en el primer periodo, pero el coste asociado potencial despido futuro hará que la empresa sea más reticente a disminuir la plantilla en el segundo periodo. Por tanto, el impacto global en el empleo resulta ambiguo, lo que dota de especial importancia al trabajo empírico. De esta forma, fundamentándonos en las líneas del modelo descrito, planteamos y estimamos, en segundo lugar, un panel dinámico con datos regionales españoles recientes, correspondientes al periodo 2005-2011. La especificación dinámica de dicho panel nos permitirá contrastar el impacto en el empleo asalariado de los costes de despido, tanto a corto como a largo plazo.

El resto del trabajo se estructurará como sigue: en el segundo apartado analizaremos el estado de la legislación sobre despidos en España, al objeto de hacer una primera aproximación al tema en nuestro país. El apartado 3 constituye el cuerpo central del trabajo. En él se plantea, en primer lugar, el marco y el modelo teórico en los que nos fundamentamos, para pasar, más tarde, al planteamiento del panel de datos y su estimación. El cuarto apartado sintetiza el trabajo y ofrece sus principales conclusiones. Por último, se incorporan dos apéndices. En el primero se sintetizan las líneas de las reformas legislativas sobre el despido en España desde 1994 a la actualidad y en el segundo se presentan las pautas seguidas en la resolución del modelo teórico.

\section{EL PANORAMA DE LOS COSTES DE DESPIDO EN ESPAÑA}

El número total de despidos de nuestro país se puede calcular sumando los datos procedentes de las siguientes estadísticas: las conciliaciones realizadas en las Unidades de Mediación, Arbitraje y Conciliación (MAC), las conciliaciones y sentencias que tienen lugar en los Juzgados de lo Social y los trabajadores afectados por Expedientes de Regulación de Empleo (Malo y Toharia, 1999). Desde 2002, año en que entra en vigor la reforma sobre prestaciones por desempleo, también podemos utilizar la suma de los siguientes datos a través de las altas de prestaciones contributivas: altas al amparo de la Ley 45/2002, altas por despidos objetivos, altas por conciliaciones en las Unidades MAC, altas mediante intervención de la jurisdicción social, altas a través de Expedientes de Regulación de Empleo, y otro tipo de altas, como por ejemplo por motivo de despido durante el periodo de prueba (Malo y Toharia, 2008) ${ }^{5}$. Este nuevo

\footnotetext{
${ }^{5}$ Como los datos sobre prestaciones por desempleo con los que contamos no nos permiten calcular las cuantías de las indemnizaciones por despido, vamos a utilizar la primera forma a lo largo del trabajo; infraestima el número total de despidos, pero nos permite calcular el coste del despido medio de cada una de las tres categorías.
} 
método para medir el número de despidos nos permite analizar el tipo de despido más utilizado a lo largo de los últimos años de crisis económica, como presentamos en el Gráfico 1. Así, la finalización de contratos temporales (valorada por separado en el eje derecho), con un coste de despido prácticamente nulo, supone la vía más utilizada durante todo el periodo 2008-2010. Esta serie está caracterizada también por una cierta estacionalidad dada la naturaleza de los contratos temporales. La segunda vía más utilizada para ajustar la plantilla es el despido exprés de la reforma $45 / 2002$, cuya ventaja principal es la rapidez en la tramitación del despido, lo que permite al empresario un ajuste prácticamente automático del factor trabajo que disminuye notablemente la incertidumbre y el tiempo del proceso de reclamación, aunque la cuantía de la indemnización sea mayor.

\section{Gráfico 1}

Altas iniciales de beneficiarios de prestaciones contributivas por causa de despido desde agosto de 2008 a noviembre de 2010

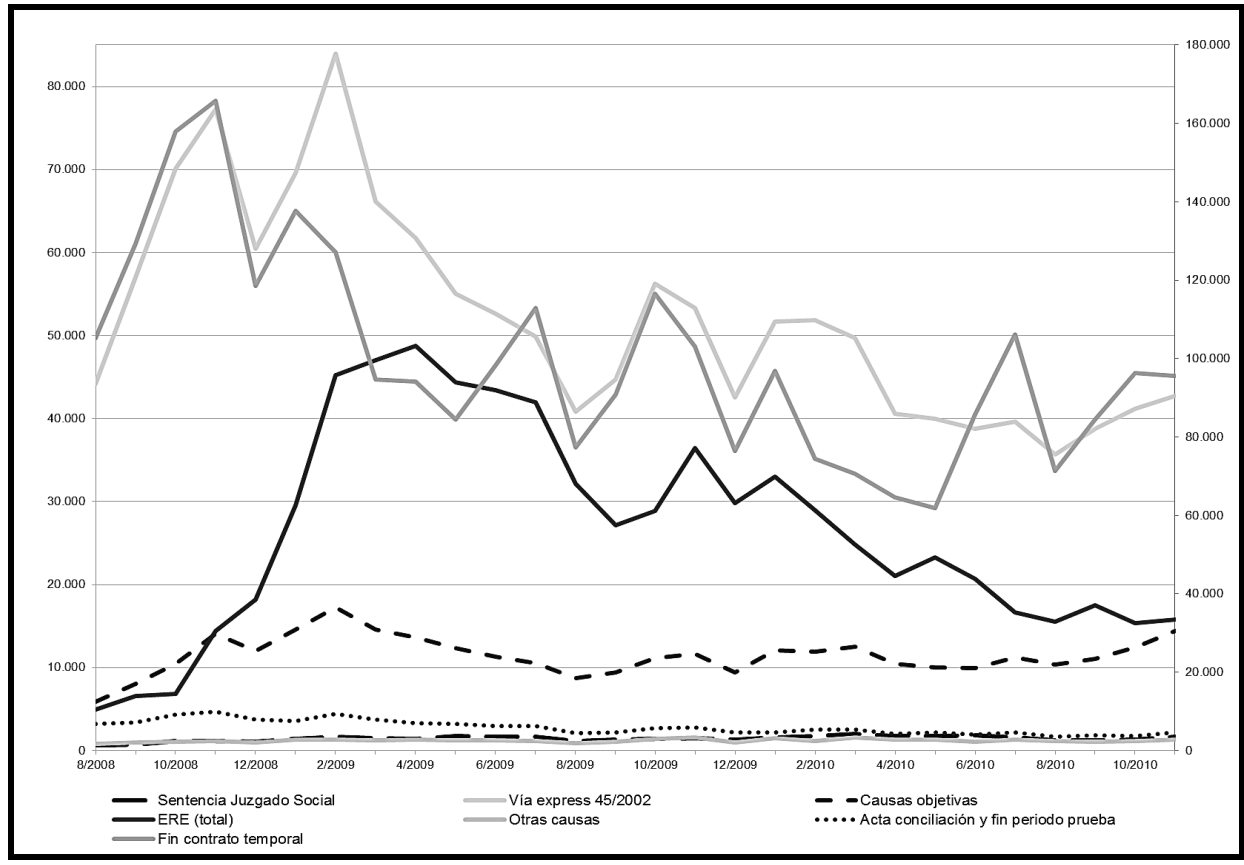

Notas: 1. En el eje derecho se miden las finalizaciones de contratos temporales; el resto de series están valoradas en el eje principal. / 2. En la modalidad de ERE se incluyen tanto Expedientes por motivo de extinción como de suspensión y desempleo parcial, para facilitar la contabilidad de los datos.

Fuente: Elaboración propia a partir del Fichero Histórico de Prestaciones por Desempleo (HSIPRE).

La tercera vía que canaliza un mayor número de despidos es la colectiva. Pese a ser más lenta que las anteriores, está más influida por la coyuntura eco- 
nómica. Los despidos por causas objetivas, contra lo que cabría esperar en una época en la que las empresas pueden tener circunstancias más ajustadas en relación con su margen de beneficios, apenas incrementan su evolución, la cual permanece además en un nivel muy bajo en relación con las otras tres vías. Esto permite concluir que, en la práctica, la legislación sobre despido no está siendo utilizada como debería, y las empresas recurren a vías menos costosas o simplemente más rápidas para ajustar su plantilla cuando lo requieren.

\section{Gráfico 2}

Despidos por asalariados privados con contrato indefinido y tasa de empleo (1994-2009)

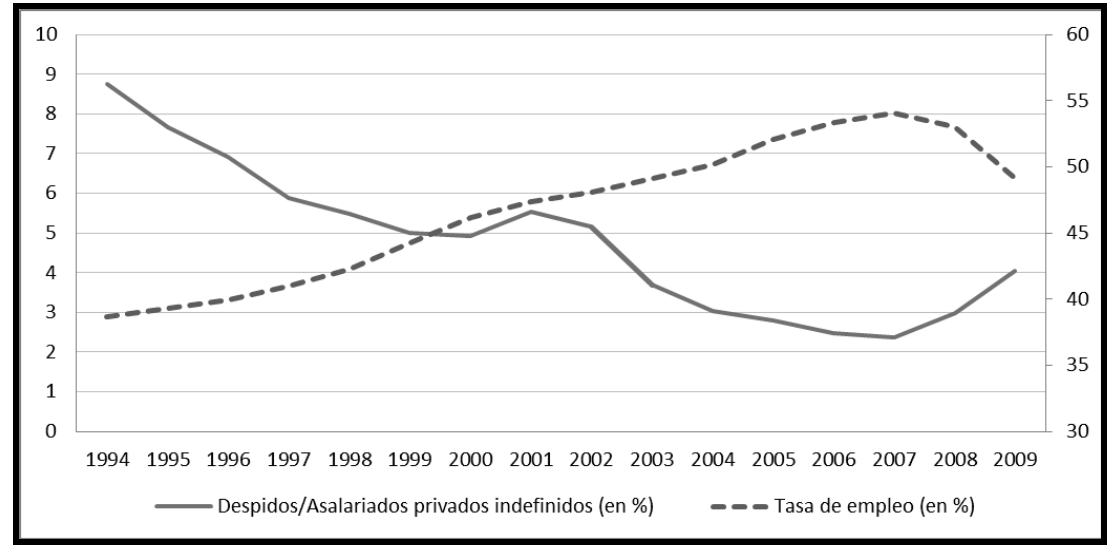

Notas: 1. En el eje derecho está medida la tasa de empleo, para poder observar mejor su variabilidad. 2. Durante el periodo 1995-1998 los datos de paro sufren una sobrevaloración debido a cambios en la metodología EPA (Malo y Toharia, 1999) / 3. Pese a que las tendencias de las series son similares, estamos dejando fuera de este análisis el número de contratos temporales no renovados, que también podrian hacer disminuir la tasa de empleo, sobre todo para los colectivos en los que se han generalizado (como el de los jóvenes).

Fuente: Elaboración propia a partir de datos del INE y del BEL.

En el Gráfico 2 comparamos la tasa de empleo media anual para el periodo 1994-2009, medida en el eje derecho, con la relación entre el número de despidos al año y aquel colectivo susceptible de ser despedido, esto es, el número de asalariados con contrato indefinido del sector privado ${ }^{6}$. El perfil de ambas series es prácticamente simétrico a lo largo del periodo, lo que indica que existe una relación inversa entre ambas variables. En el periodo de 2000 a 2002 pode-

\footnotetext{
${ }^{6}$ Pese a que Malo y Toharia (1999) no obtienen el número de asalariados del sector privado con contrato indefinido para su periodo de estudio (1978-1998), a través de la correspondencia entre asalariados con contrato indefinido y asalariados del sector privado, hemos podido disponer de datos actualizados que sí cuentan con la variable asalariados del sector privado según tipo de contrato o relación laboral. Excluye igualmente a los trabajadores con contrato temporal, cuyo coste de despido es prácticamente nulo, y a los trabajadores del sector público, cuya mayor parte a efectos prácticos no son susceptibles de ser despedidos.
} 
mos observar cómo el repunte del número de despidos con relación a los asalariados no se tradujo en una reducción de la tasa de empleo ya que ésta permanece con tendencia ascendente.

Esta relación, a su vez, tiene que ver con el ciclo económico; en la medida en que el peso del número de despidos sobre los asalariados aumenta, la tasa de empleo se reduce. Así, durante la primera fase del periodo, caracterizada por la crisis económica de los primeros años noventa, la tasa de empleo se encuentra en su nivel más bajo, en torno al $40 \%$, y la relación entre despidos por asalariados es superior al 5\%. Igualmente, a partir de 2007, con la irrupción de la reciente crisis económica, la tasa de empleo vuelve a descender llevada por un incremento de la tasa de despidos sobre asalariados. La relación entre ambas variables, además de ser inversa, tiene que ver con el fenómeno que Malo y Toharia (1999) denominan comportamiento "adelantado" del número de despidos respecto de la tasa de paro ${ }^{7}$. Así, en la medida en que se produce un despido, la tasa de paro se incrementa con ligero rezago. Este hecho también se puede observar en la evolución de las series de nuestro gráfico. En la última parte del trabajo trataremos de justificar la relación inversa entre estas variables en función del coste de despido.

Analizando la composición del coste laboral por trabajador podemos comprobar cómo existen diferencias sectoriales en relación con el coste de despido. La Encuesta Anual de Coste Laboral (ECLab) de 2010, que aun a pesar de lo dicho por Malo y Toharia (1999) acerca de sus deficiencias, nos puede aportar información a grandes rasgos sobre dichas discrepancias y una visión amplia sobre la distribución porcentual de los costes. En el Cuadro 1 podemos observar cómo efectivamente la indemnización por despido anual en la industria es superior a la del sector de la construcción, y sobre todo, al de los servicios.

De nuevo es importante remarcar que las indemnizaciones por finalización de contrato no se incluyen en el coste de despido. Se observa que, en total, las indemnizaciones por despido constituyen el $1,3 \%$ del coste total bruto de un trabajador, porcentaje que se eleva ligeramente si lo comparamos con el coste total neto.

El Instituto Nacional de Estadística, en este caso a través de la Encuesta Trimestral de Coste Laboral (ETCL), elabora una serie llamada "coste de despido". Según la metodología de la ETCL, esta variable representa toda aquella percepción que recibe por el trabajador en concepto de indemnización por despido, e incluye los salarios de tramitación y excluye los pagos por finalización de contrato temporal. En la evolución de esta variable se produce un notable efecto estacional, como consecuencia de la incorporación de las pagas

\footnotetext{
${ }^{7}$ Hay que tener en cuenta que en el gráfico hemos representando la tasa de empleo. Sin embargo, replicando el ejercicio para la tasa de paro podríamos ver igualmente el efecto descrito.
} 
extraordinarias en la composición de la muestra. Este fenómeno lo vamos a tratar de corregir elaborando un índice que será el resultado de dividir la serie entre el coste laboral total, lo que nos permitirá analizar la presión que tiene el coste de despido sobre los costes de personal por trabajador.

\section{Cuadro 1}

Composición del coste laboral en los distintos sectores en 2010 (en euros)

\begin{tabular}{|c|c|c|c|c|}
\hline & Total & Industria & Construcción & Servicios \\
\hline COSTE BRUTO ${ }^{1}$ & $30.819,53$ & $35.039,81$ & $30.816,23$ & $29.931,24$ \\
\hline Sueldos y salarios & $22.540,83$ & $25.123,08$ & $21.663,33$ & $22.102,21$ \\
\hline Cotizaciones obligatorias & $6.806,61$ & $7.851,75$ & $7.497,33$ & $6.503,04$ \\
\hline Cotizaciones voluntarias & 217,04 & 329,52 & 182,40 & 198,60 \\
\hline Prestaciones sociales directas & 322,70 & 412,51 & 119,85 & 328,25 \\
\hline Gastos de carácter social & 41,65 & 63,42 & 14,93 & 40,43 \\
\hline Indemnizaciones por despido & 412,29 & 819,78 & 418,62 & 325,38 \\
\hline Gastos en formación profesional & 104,34 & 128,17 & 55,17 & 105,34 \\
\hline Gastos en transporte & 18,05 & 50,05 & 29,10 & 10,35 \\
\hline Resto de costes ${ }^{2}$ & 356,02 & 261,53 & 835,50 & 317,64 \\
\hline Subvenciones y deducciones & 278,25 & 362,34 & 239,83 & 264,86 \\
\hline COSTE NETO ${ }^{3}$ & $30.541,28$ & $34.677,47$ & $30.576,40$ & $29.666,38$ \\
\hline
\end{tabular}

Notas: 1. Excluye dietas y gastos de viaje / 2. Indemnizaciones por fin de contrato, pagos compensatorios, pequeño utillaje, ropa de trabajo, selección de personal... / 3. Deducidas subvenciones y deducciones.

Fuente: INE.

En el Gráfico 3 se presenta el promedio de dicho índice de coste de despido por Comunidad Autónoma, en el que centraremos las estimaciones de la siguiente parte del trabajo. Las Comunidades Autónomas con una mayor presión del coste de despido sobre el coste laboral son Extremadura, Canarias, Galicia, Castilla-La Mancha y Murcia, que supera la media del 1,3\%. A su vez, existe una serie de Comunidades que presenta un índice más bajo, en torno al valor 1\%. Éstas son Madrid, Cataluña, Navarra y País Vasco. Pese a que la legislación sobre despidos sea común en toda España, observamos también que existen notables diferencias de media entre unas Comunidades y otras, y además, hemos de tener en cuenta que hay marcados efectos regionales, quizá no tanto en relación con el propio coste de despido, sino en otras variables que influyen sobre él mismo, como por ejemplo la tasa de temporalidad, el número de asalariados en relación con la población potencialmente activa y la tasa de paro.

La heterogeneidad existente entre unas regiones y otras no hace sino agrandar la problemática existente derivada de una legislación común del despido. Así pues, nos sometemos a un mercado de trabajo fragmentado que mientras no 
resuelva o, al menos, intente minimizar dichas diferencias internas, los beneficios se verán menguados y los perjuicios latentes en el mercado de trabajo se acentuarán. Es por esto por lo que conviene realizar un análisis empírico que tenga en cuenta estas diferencias regionales, sobre todo en relación con la estructura del empleo, que diverge altamente entre distintas Comunidades Autónomas.

\section{Gráfico 3}

Promedio del índice de coste de despido sobre el coste laboral total durante el periodo 2005-2011 y por Comunidades Autónomas (en \%)

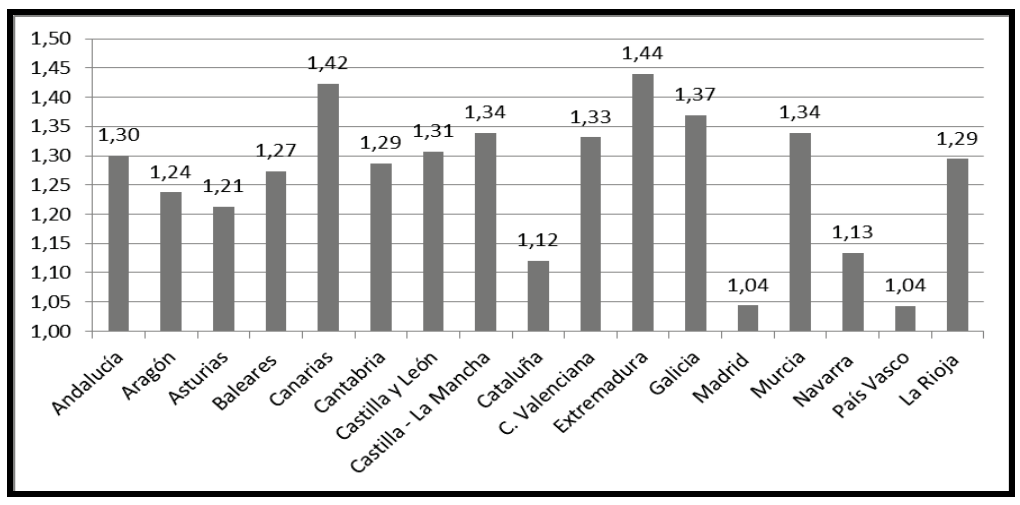

Nota metodológica: el índice se ha elaborado dividiendo el coste de despido medio por trabajador entre el coste laboral total medio por trabajador para cada Comunidad Autónoma, y representa la presión que el coste de despido produce en el coste laboral medio de cada trabajador. En el siguiente apartado profundizaremos en una fundamentación más exhaustiva de dicho índice.

Fuente: Elaboración propia a partir de datos de la ETCL (INE).

\section{ESTIMACIÓN DEL EFECTO DE LOS COSTES DE DESPIDO SOBRE EL EMPLEO EN ESPAÑA}

\subsection{Marco teórico}

La primera reflexión que cabe hacerse al intentar modelizar el comportamiento del mercado de trabajo en España es si las herramientas habituales del análisis económico (básicamente la oferta y la demanda) continúan teniendo validez a la hora de fijar los salarios y el nivel de empleo. Como señala Sánchez Molinero (1992), es norma habitual en muchos países (incluido el nuestro) que los salarios se fijen mediante un mecanismo de negociación colectiva en el que, idealmente, estarían implicados dos sindicatos, uno laboral y otro de empresarios. Es cierto que el salario ya no se fijaría mediante un cúmulo de negociaciones bilaterales, sino mediante una única negociación a escala multilateral en la que primarían especialmente los argumentos de presión que cada uno de los dos 
grupos pueda aducir. No obstante, las funciones de oferta y demanda de trabajo seguirían existiendo, y tras de ellas se seguirían escondiendo las decisiones de un amplio número de agentes individuales y optimizadores que, además, continuarían aceptando el salario negociado y las posibles restricciones normativas como datos. ${ }^{8}$

La presencia de costes de despido se legitima, en primera instancia, desde un punto de vista de justicia social, al servir como mecanismo de protección del trabajador frente a despidos injustificados por parte de la empresa. Si, en el ámbito de la teoría de los contratos implícitos, empresas y trabajadores individuales negociasen la cuantía de estas indemnizaciones como un elemento más del contrato laboral, las mismas quedarían internalizadas y no tendrían por qué apreciarse distorsiones en el equilibrio. ${ }^{9}$ Cuando dichas indemnizaciones son fijadas de forma centralizada, más allá de la negociación bilateral entre agentes, su presencia implicaría una elevación del coste laboral esperado y, por tanto, tendría repercusiones inmediatas en la firma de nuevos contratos de empleo por las empresas. No obstante, la existencia de un coste despido que debe satisfacerse al trabajador en el momento de rescindir su contrato laboral, hará que la empresa sea más reticente al despido de lo que sería en ausencia de dicha indemnización. Así pues, desde una perspectiva dinámica y a largo plazo, el efecto global del coste despido sobre el empleo medio no está claramente determinado, pudiendo llegar a ser completamente neutral en muchas de las especificaciones teóricas utilizadas.

El modelo que nosotros proponemos sigue las líneas de Bentolila y SaintPaul (1994) y Eeckhoudt y Treich (2003) y pretende recoger las reflexiones llevadas a cabo en el párrafo anterior. Supondremos una empresa neutral al riesgo que toma decisiones de contratación de un input $(l)$ en dos periodos de tiempo ( 1 y 2 ) sin factor de descuento. En el momento 1 la empresa toma su decisión de contratación de input $\left(l_{1}\right)$ conociendo el estado actual de la economía. No obstante, la empresa desconoce aún el estado de la economía del momento futuro. En el momento 2, y según sea la realización del shock exógeno $(\tilde{\varepsilon})$, la empresa toma su nueva decisión de contratación de trabajo $\left(l_{2}\right)$.

Supondremos que si el volumen de empleo del periodo 2 es menor que el nivel de empleo contratado en el periodo previo $\left(l_{2}<l_{1}\right)$, el ajuste necesario

\footnotetext{
${ }^{8}$ Simón (2001) considera, no obstante, que "la estructura de salarios pactados presenta características dificilmente conciliables con un enfoque competitivo del mercado de trabajo, tales como la elevada influencia que ejercen en la determinación de los salarios pactados factores como la rama de actividad y el territorio de referencia de los convenios, o la duración de la jornada de trabajo".

${ }^{9}$ Este es el argumento de Lazear (1990), también apuntado, entre otros, por Dolado y Jimeno (2004).
} 
implicará un coste de despido que es positivo y creciente. El problema de la empresa sería entonces:

$$
\max _{l_{1}}\left\{B\left(l_{1}\right)+\left\{\max _{l_{2}} E\left[B\left(l_{2}, \tilde{\varepsilon}\right)-\delta \cdot g\left(l_{1}-l_{2}\right)\right]\right\}\right\}
$$

donde $B\left(l_{1}\right)$ y $B\left(l_{2}, \tilde{\varepsilon}\right)$ representan las ecuaciones de beneficios de los periodos 1 y 2 , respectivamente. El término $\tilde{\varepsilon}$ indica un shock exógeno de demanda o productividad. Por su parte, $\delta \cdot g\left(l_{1}-l_{2}\right)$ representa la función de costes de ajuste (a la baja) del input trabajo, en donde $\delta$ es un parámetro positivo y $g\left(l_{1}-l_{2}\right)$ una función nula cuando $l_{2} \geq l_{1}$ y positiva y creciente cuando $l_{2}<l_{1}$ :

$$
\delta>0
$$

$$
g\left(l_{1}-l_{2}\right)= \begin{cases}0 & \text { si } l_{2} \geq l_{1} \\ g\left(l_{1}-l_{2}\right)>0 ; g^{\prime}>0 ; & \text { si } l_{2}<l_{1}\end{cases}
$$

El nivel de empleo intertemporal, en función de $\delta$, será:

$$
l(\delta)=l_{1}(\delta)+E\left\{l_{2}\left[l_{1}(\delta), \tilde{\varepsilon}, \delta\right]\right\}
$$

Y nos interesa averiguar cómo se ve afectado si se modifica el parámetro $\delta$ :

$$
l^{\prime}(\delta)=\underbrace{\frac{d l_{1}(\delta)}{d \delta}}_{(<0)}+\underbrace{\frac{d E\left[l_{2}\left(l_{1}(\delta), \tilde{\varepsilon}, \delta\right)\right]}{d \delta}}_{\text {(ambiguo) }}
$$

donde:

$$
\frac{d E l_{2}^{*}}{d \delta}=\underbrace{\frac{\partial E l_{2}^{*}}{\partial \delta}}_{(>0)}+\underbrace{\underbrace{\frac{\partial E l_{2}^{*}}{\partial l_{1}}}_{(<0)}}_{(<0) \text { sig convexa }} \cdot \underbrace{\frac{d l_{1}^{*}}{d \delta}}_{(<0) v e x a}
$$

Puede demostrarse que:

1) De manera no-ambigua, el establecimiento (o aumento) de un coste de despido hará más costoso un presunto ajuste futuro, por lo que la empresa tenderá a reducir el nivel de contratación presente de trabajo.

$$
\frac{d l_{1}(\delta)}{d \delta}<0
$$

2) No obstante, el efecto del establecimiento (o aumento) de un coste de despido sobre el nivel esperado de empleo futuro resulta ambiguo, pudiendo ser incluso positivo.

$$
\frac{d E l_{2}^{*}}{d \delta}<0
$$


a. Por un lado, opera un efecto directo. Dado un determinado volumen de trabajo contratado en el periodo previo $\left(l_{1}^{*}\right)$, y ante una coyuntura adversa, un ajuste a la baja de la plantilla (en el periodo 2) involucra un nuevo coste marginal (el de despido), lo que hará que la empresa sea más reticente a reducir el tamaño de su plantilla.

$$
\frac{\partial E l_{2}^{*}}{\partial \delta}>0
$$

b. Pero también existe, por otro lado, un efecto indirecto asociado al hecho de que el volumen esperado de empleo del periodo 2 depende del contratado previamente en el periodo 1. Este efecto tendría signo negativo si la función de ajuste $g$ es convexa.

$$
\frac{\partial E l_{2}^{*}}{\partial l_{1}} \cdot \frac{d l_{1}^{*}}{d \delta}<0 ;(\text { con } g \text { convexa) }
$$

En resumen, nuestro modelo predice que un establecimiento (revisión) de un coste de despido tiene, inequívocamente, un efecto negativo sobre el nivel de empleo a corto plazo. No obstante, el efecto total acumulado a largo plazo es ambiguo, pudiendo llegar a ser inapreciable. Estas son las hipótesis básicas que pretendemos contrastar a continuación.

\subsection{Especificación funcional}

De acuerdo con lo expuesto en el apartado teórico previo, vamos a especificar una ecuación de empleo que pretende ser una forma reducida del funcionamiento de un modelo estructural de mercado de trabajo:

$$
L=f(C D, W, \mathbf{D}, \mathbf{X})
$$

en donde $L$ es la medida relativa del empleo susceptible de ser despedido; $C D$ representa la variable explicativa fundamental y se trata de un índice que recoge el coste de despido relativo por trabajador; por su parte, la variable $W$ representa el coste laboral total por trabajador en términos reales. Para que la ecuación de empleo esté correctamente especificada se incorpora, además, los vectores D y $\mathbf{X}$ que recogen otro tipo de factores exógenos que podrían desplazar, respectivamente, las curvas de demanda y oferta de trabajo que esconde tras de sí la forma reducida.

Para poder captar el impacto sobre el empleo de los costes de despido tanto a corto como a largo plazo la especificación econométrica concreta de nuestro modelo será la siguiente:

$$
L_{i t}=\mu+\gamma L_{i t-1}+\beta_{0} C D_{i t}+\beta_{1} C D_{i t-1}+\delta \mathbf{D}_{i t-1}^{\prime}+\lambda \mathbf{X}_{i t-1}^{\prime}+v_{i t}
$$


Se trata de un panel de datos en donde $i$ hace referencia a la unidad espacial (Comunidades Autónomas) y $t$ a los periodos de tiempo (trimestres). Por su parte, $v_{i t}$ es el término de perturbación, que se puede descomponer como $v_{i t}=\mu_{i}$ $+\varepsilon_{i t}$, donde $\mu_{i}$ es una componente específica a la región y $\varepsilon_{i t}$ es un término puramente aleatorio. El desfase en la variable dependiente y en el regresor fundamental $(C D)$ dotan al panel del carácter dinámico necesario para contrastar nuestras hipótesis. Por último, los vectores de variables que recogen los shocks de demanda y de oferta (D y S) se incorporan al modelo de forma desfasada para garantizar su carácter exógeno. La especificación funcional adoptada es doble-logarítmica, por lo que los coeficientes obtenidos en las estimaciones son, directamente, elasticidades. ${ }^{10}$

Para la estimación de este tipo de modelos, si $T$ es suficientemente grande, se puede utilizar el estimador intragrupos ya que es consistente (Nickell, 1981). En nuestro caso resulta más adecuado el uso del Método Generalizado de los Momentos (GMM) de acuerdo con las líneas de Arellano-Bond (1991), ArellanoBover (1995) y Blundell-Bond (1998). ${ }^{11}$ Para ello, se estima el modelo presentado en primeras diferencias ${ }^{12}$ utilizando los retardos de las variables endógenas como instrumentos.

\subsection{Variables utilizadas y fuentes estadísticas}

Como ya hemos indicado, todas las variables del modelo son logarítmicas por lo que, a continuación omitiremos referirnos a este hecho. La variable dependiente $(A S A P)$ es la proporción de trabajadores por cuenta ajena sobre el total de la población en edad de trabajar. Este dato proviene de la Encuesta de Población Activa (EPA) del Instituto Nacional de Estadística (INE).

La variable explicativa fundamental, esto es los costes de despido, ha sido aproximada mediante dos índices alternativos con el objeto de apreciar mejor la

${ }^{10}$ La especificación doble-logarítmica resulta una práctica bastante habitual en otros contextos que también estiman ecuaciones de empleo. Tal es el caso, por ejemplo, de la estimación de los efectos de los salarios mínimos sobre el empleo, fenómeno que cuenta con una abultada literatura. No obstante, utilizando esta especificación estamos suponiendo, implícitamente, que el impacto porcentual sobre el empleo de un cambio porcentual en los costes despido (esto es, la elasticidad) es constante, algo que podría no ser cierto. Para contrastar la validez de nuestra especificación hemos llevado a cabo estimaciones alternativas en las que el coste de despido aparece tanto en forma lineal como cuadrática, al objeto de averiguar si el impacto sobre el nivel de empleo va acelerándose (o decelerándose) al variar el nivel de los costes de despido relativos. En los resultados obtenidos, los coeficientes de los términos cuadráticos no resultan ser significativos, dando validez a nuestra especificación inicial del modelo. Las estimaciones están disponibles bajo petición a los autores.

${ }^{11}$ Una excelente revisión de estos modelos puede encontrarse en Arellano y Honoré (1999).

${ }^{12}$ Lo que, además, garantiza la estacionariedad de los regresores. 
estabilidad de su impacto a la especificación adoptada. Por un lado, se ha empleado $C D$-ratio, esto es, el cociente entre los costes de despido y el coste laboral total $(C L)$; y, por otro lado, se han empleado los costes de despido por trabajador en términos reales (CD-real). Los datos provienen de la Encuesta Trimestral de Coste Laboral del INE.

Como control de la influencia del ciclo económico en la demanda de trabajo (shocks de demanda) se ha empleado la tasa de paro de los prime-age males o varones en edad principal (entre 30 y 45 años) (UPAV). Se trata de un variable utilizada con gran profusión en este tipo de estimaciones para captar (en forma inversa) el impacto del ciclo sobre diferentes formas de ocupación relativa. ${ }^{13}$ Los datos provienen de la EPA.

Todas las variables de oferta se han obtenido de la EPA y pueden agruparse en dos categorías:

En primer lugar, un conjunto de variables que pretenden controlar las posibles variaciones exógenas en la fuerza de trabajo. Para ello se han empleado los pesos que suponen las diferentes cohortes laborales de sexo y edad sobre la población laboral total (mayores de 16 años) ${ }^{14}$. Estos ratios, a los que denominaremos $R P O B(s, e)$, se definirán para ambos sexos ( $s=$ varón, mujer) y tres grupos de edad (e=16-19, 20-24, y más de 55 años). ${ }^{15}$

En segundo lugar, se ha controlado también la composición de la fuerza laboral mediante la inclusión de las dos siguientes variables. Por un lado, la tasa de empleo agrario (TEAGR), como control del peso relativo de dicho sector en cada región, intentando amortiguar el hecho de que las variables salariales utilizadas se refieran a la industria, a la construcción o a los servicios. Por otro lado, la tasa de temporalidad (TEMP) o relación entre el número de asalariados con contrato temporal sobre el total de asalariados. Incluimos esta variable en la estimación dado que los contratos temporales presentan un coste de despido prácticamente nulo.

Además de las variables descritas también se han incorporado controles estacionales del nivel de empleo.

${ }^{13}$ Es habitual utilizar, para captar el impacto del ciclo, como alternativa a esta variable la tasa de variación del valor añadido bruto real de cada Comunidad Autónoma. No obstante, los datos de esta última referentes a los últimos periodos que incorporamos en las estimaciones no se encuentran aún disponibles. Es por ello que en las estimaciones solamente incorporemos como control cíclico la variable UPAV.

${ }^{14}$ Las tasas de actividad podrían constituir una mejor aproximación de los cambios en la oferta de trabajo, no obstante adolecerían de un marcado carácter endógeno.

${ }^{15}$ Con el objeto de no incurrir en la "trampa de las variables ficticias" se adoptan como referencia los grupos de edad principal (25-54 años) para ambos sexos. 
Después de haber definido las variables, los modelos de panel que vamos a estimar adoptan la siguiente especificación concreta:

$$
A S A P_{i t}=f\left(C D-r_{i t}, C L_{i t}, U P A V_{i t}, T E A G R_{i t}, T E M P_{i t}, \operatorname{RPOB}(s, e)_{i t}\right)
$$

donde el subíndice $i$ representa la región correspondiente, excluyendo Ceuta y Melilla, $(i=1, \ldots, 17), t$ el trimestre dentro del periodo 2005-2011 $(t=1, \ldots, 28)$. Por su parte, el índice de costes de despido $(C D)$ adopta dos especificaciones $(r=$ ratio, real), y los ratios de población $(R P O B)$ se refieren a ambos sexos y a las tres cohortes de edad descritas previamente.

Los descriptivos (en niveles) de las principales variables utilizadas en la estimación de presentan en el Cuadro 2. Los datos hacen referencia a todo el periodo analizado y el conjunto del país.

\section{Cuadro 2}

Estadísticos descriptivos

\begin{tabular}{ccccc}
\hline España & Media & Máximo & Mínimo & Desviación estándar \\
\hline ASAP & 0,333 & 0,453 & 0,210 & 0,051 \\
CD-real & 27,544 & 52,087 & 14,434 & 8,827 \\
CD-ratio & 0,013 & 0,027 & 0,006 & 0,004 \\
CL & 2187,65 & 2838,79 & 1707,05 & 235,68 \\
UPAV & 0,094 & 0,263 & 0,008 & 0,062 \\
TEMP & 0,292 & 0,473 & 0,169 & 0,066 \\
\hline
\end{tabular}

Fuente: Elaboración propia.

\subsection{Resultados}

En el Cuadro 3 presentamos los resultados de las estimaciones de los paneles dinámicos en sus dos especificaciones: utilizando como variable dependiente fundamental el coste de despido en términos reales ( $C D$-real), como en forma de ratio sobre el coste laboral total por trabajador (CD-ratio). La consistencia del estimador GMM depende del cumplimiento de los supuestos de ausencia de correlación serial en el término de error y de validez de los instrumentos utilizados. Para contrastar la primera de esas hipótesis se han aplicado los tests de Arellano y Bond (1991) para los procesos AR(1) y, esencialmente, AR(2) en primeras diferencias. En ambos casos los p-valores obtenidos confirman la validez de las dos especificaciones que utilizamos. En el caso del contraste de validez de los instrumentos, se ha utilizado el test de Sargan propuesto en Arellano y Bover (1995), cuya hipótesis nula es la exogeneidad de los instrumentos como grupo. Los p-valores obtenidos muestran que no podemos rechazar la validez de los instrumentos a los niveles de significación habituales. Una vez 
comprobada la validez econométrica de nuestra especificación pasaremos a comentar los principales resultados obtenidos.

En cuanto a los coeficientes estimados, observamos cómo la variable dependiente fundamental, que representa el impacto contemporáneo de los costes de despido, presenta en ambos modelos estimados sendos coeficientes con signos negativos y altamente significativos. Los retardos de dichos coeficientes, también han resultado significativos en ambas especificaciones presentando, en este caso, signos positivos.

\section{Cuadro 3}

Resultados de las estimaciones

(Variable dependiente ASAP $_{t}$ )

\begin{tabular}{|c|c|c|}
\hline Regresores & (CD-real) & (CD-ratio) \\
\hline ASAP $_{t-1}$ & $0,908^{\star \star *}$ & $0,901^{\star \star *}$ \\
\hline $\mathrm{CD}_{\mathrm{t}}$ & $-0,064^{* \star *}$ & $-0,057^{\star \star *}$ \\
\hline $\mathrm{CD}_{\mathrm{t}-1}$ & $0,043^{* *}$ & $0,042^{* *}$ \\
\hline $\mathrm{CL}_{\mathrm{t}-1}$ & $-0,030$ & 0,044 \\
\hline UPAV $_{t-1}$ & 0,0007 & 0,002 \\
\hline TEMP $_{t-1}$ & $-0,026^{*}$ & $-0,022$ \\
\hline TEAGR $_{\mathrm{t}-1}$ & $-0,005^{\star}$ & $-0,003^{*}$ \\
\hline RPOB(VAR,16-19) $)_{t-1}$ & $0,372^{*}$ & $0,388^{*}$ \\
\hline RPOB(VAR,20-24)-1 & $-0,105$ & $-0,121$ \\
\hline RPOB $(\text { VAR, },+54)_{t-1}$ & 0,056 & 0,074 \\
\hline$R P O B(M U J, 16-19)_{t-1}$ & $-0,442^{*}$ & $-0,475^{\star}$ \\
\hline RPOB(MUJ,20-24)-1 & $0,183^{*}$ & $0,219^{*}$ \\
\hline RPOB $(M U J,+54)_{t-1}$ & $-0,064$ & $-0,084$ \\
\hline Constante & $-0,084$ & $-0,158^{\star *}$ \\
\hline $\mathbf{N}$ & 459 & 459 \\
\hline Test Arellano-Bond AR(1) & $\begin{array}{c}z=-3,06 \\
\operatorname{Pr}>z=0,002\end{array}$ & $\begin{array}{c}z=-2,98 \\
\operatorname{Pr}>z=0,003\end{array}$ \\
\hline Test Arellano-Bond AR(2) & $\begin{array}{c}z=-1,09 \\
\operatorname{Pr}>z=0,278\end{array}$ & $\begin{array}{c}z=-1,09 \\
\operatorname{Pr}>z=0,279\end{array}$ \\
\hline Test Sargan & $\begin{array}{c}\text { chi2 }=424,33 \\
\operatorname{Pr}>\text { chi2 }=0,244\end{array}$ & $\begin{array}{c}\operatorname{chi} 2=430,35 \\
\operatorname{Pr}>\operatorname{chi} 2=0,185\end{array}$ \\
\hline
\end{tabular}

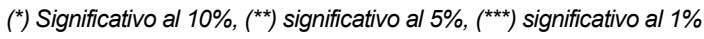

Fuente: Elaboración propia.

Para hacer una mejor valoración de estos resultados, y aprovechando la especificación dinámica de nuestro panel, ofrecemos en el Cuadro 4 los multi- 
plicadores de impacto o contemporáneos y los totales o de largo plazo para ambas especificaciones del coste de despido.

\section{Cuadro 4}

Multiplicadores de impacto y totales resultantes de las estimaciones

\begin{tabular}{lcc}
\hline & Multiplicador de impacto $(\mathbf{c} / \mathbf{p})^{(1)}$ & ${\text { Multiplicador total }(\mathbf{l} / \mathbf{p})^{(2)}}$ \\
\hline CD-real & $-0,064^{* * *}$ & $-0,232$ \\
CD-ratio & $-0,057^{* \star *}$ & $-0,158$ \\
\hline
\end{tabular}

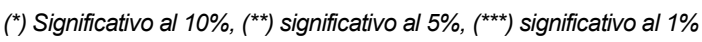

(1): Los multiplicadores de impacto son los coeficientes contemporáneos de las variables de CD. Su significación se obtiene a partir los correspondientes estadísticos t obtenidos directamente en las estimaciones.

(2): Los multiplicadores totales se obtienen teniendo en cuenta la especificación tipo $A D R L(1,1)$ del modelo, operando con los coeficientes de las variables de coste de despido (contemporánea y desfasada) y el de la endógena desfasada de la forma siguiente: $\left.\left[C D(r)_{t}+C D(r)_{t-1}\right)\right] \cdot\left(1-A S A P_{t-1}\right)^{-1}$. Su significación estadística se obtiene a través de sendos tests de Wald sobre combinaciones no lineales de coeficientes. En el caso del modelo con CD-real, el p-valor obtenido para la elasticidad de largo plazo es de 0,224 y en el caso del modelo con CD-ratio de 0,380.

Fuente: Elaboración propia.

Las elasticidades de impacto obtenidas en ambos modelos son, como hemos visto, estadísticamente significativas y, además, sus magnitudes son bastante similares. De esta forma, podemos afirmar que, según el modelo con $C D$-real, un aumento de un 10\% de los costes de despido sobre el IPC en un cierto periodo, reduciría el empleo relativo asalariado de ese mismo periodo en algo más del $0,6 \%$. Por otro lado, según el modelo con CD-ratio, un aumento de un $10 \%$ del peso que los costes de despido suponen sobre los costes totales en un cierto periodo, reduciría el empleo relativo asalariado de ese mismo periodo en algo menos del $0,6 \%$.

Una forma de apreciar mejor la magnitud de este impacto, consiste en compararla con las elasticidades de corto plazo que se han provisto en las estimaciones sobre el efecto del aumento relativo del Salario Mínimo Interprofesional (SMI) en el empleo relativo de los trabajadores más jóvenes en España. Esto es así porque, en ambos casos, se trata de calibrar el impacto del cambio de un coste relativo sobre un nivel de empleo relativo. En este sentido, los diversos trabajos realizados para España ${ }^{16}$ nos informan de que un aumento de un $10 \%$ del SMI (con relación al salario medio) reduce el empleo de los más jóvenes (en relación a su población laboral) entre un 1,2 y un 6\%. Así pues, en relación a esta referencia, el impacto contemporáneo del aumento relativo en los costes de despido es, en valor absoluto, apreciablemente menor.

\footnotetext{
${ }^{16}$ Entre la amplia literatura existente al respecto, puede consultarse el reciente trabajo de González et al. (2012).
} 
La especificación dinámica del modelo nos permite calcular también las elasticidades (multiplicadores) totales para ambos modelos estimados. Como resulta conocido, este multiplicador nos informaría del efecto acumulado, o a largo plazo, del cambio en un regresor (en nuestro caso, los costes de despido) sobre la variable dependiente (el empleo asalariado relativo). En ambos modelos estimados, los multiplicadores totales obtenidos no resultan ser estadísticamente distintos de cero. Este resultado nos permite afirmar que el impacto negativo sobre el empleo que a corto plazo detectábamos a raíz de un aumento en los costes de despido relativos termina difuminándose con el paso del tiempo resultando, a largo plazo, un efecto total inapreciable.

Estos resultados encajan dentro de las posibilidades que abre el modelo teórico previamente planteado y, además, encajan en las predicciones habituales de los modelos dinámicos de demanda de trabajo.

\section{CONCLUSIONES}

En este trabajo hemos analizado la relación existente entre los costes de despido y el nivel de empleo de nuestro país.

En lo referente a la medición de los costes de despido, hemos visto cómo uno de los problemas al que se enfrenta su análisis en nuestro país es la escasa y poco fiable información estadística disponible sobre la materia. Además de la carencia de fuentes de datos, las existentes tienen múltiples deficiencias y no son homogéneas, lo que nos impide estandarizar el análisis acerca del número de despidos y de las indemnizaciones reales.

En la parte central del trabajo, hemos propuesto un modelo teórico de dos periodos con incertidumbre. Según este modelo, la presencia de futuros costes de despido volverá al empresario más cauto a la hora de contratar nuevos trabajadores en el primer periodo; pero, en cambio, el coste asociado al despido hará que la empresa sea más reticente a disminuir la plantilla en el segundo periodo. De esta forma, el efecto total o a largo plazo de la implantación o aumento de un coste de despido resulta ambiguo, pudiendo ser incluso despreciable, lo que dota de gran relevancia a la investigación empírica. Para este fin, hemos elaborado y estimado un panel dinámico con datos de las distintas Comunidades Autónomas españolas durante los diferentes trimestres del periodo 2005-2011.

En las estimaciones se ha utilizando como variable dependiente fundamental, alternativamente, el coste de despido en términos reales y el ratio de estos costes sobre el coste laboral total por trabajador. En cualquiera de los dos casos, hemos comprobado que un aumento de un $10 \%$ en la variable de coste de despido en un cierto periodo reduciría el empleo relativo asalariado de ese mismo periodo en torno a un $0,6 \%$. Se trata, por tanto, de un impacto negativo y altamente significativo desde el punto de vista estadístico, aunque bastante moderado en valor absoluto. 
Por su parte, las elasticidades totales que hemos obtenido han resultado ser indistinguiblemente distintas de cero, con independencia de la especificación utilizada. Este resultado nos permite afirmar que el impacto negativo sobre el empleo que a corto plazo detectábamos a raíz de un aumento en los costes de despido termina difuminándose con el paso del tiempo resultando, a largo plazo, un efecto total inapreciable. Se trata de un resultado que concuerda con las predicciones habituales de los modelos dinámicos de demanda de trabajo.

Aunque no resulta posible realizar una valoración rigurosa de los efectos de la última reforma laboral (2012), sí que está claro que tendrá efectos notables sobre las vías utilizadas para despedir y sobre el coste medio que estos despidos suponen. Ante dicha reducción cabría esperar, de acuerdo con las estimaciones previas, un impacto positivo a corto plazo sobre el empleo, aunque moderado y, posiblemente, incapaz de consolidarse a largo plazo. Debe tenerse en cuenta, además, que mientras las expectativas de crecimiento sean adversas ese potencial incremento a corto plazo de nuevos contratos puede verse, en gran medida, contrapesado por la resolución, más barata, de otros contratos firmados en épocas de bonanza pasadas.

\section{REFERENCIAS BIBLIOGRÁFICAS}

ARELLANO, M. y S. BOND (1991): "Some Tests of Specification for Panel Data: Monte Carlo. Evidence and an Application to Employment Equations", en Review of Economic Studies, vol. 58, pp. 277-297.

ARELLANO, M. y BOVER. O. (1995): "Another look at the instrumental variable estimation of error-components models", en Journal of Econometrics, Vol. 68 (1), pp. 29-51.

ARELLANO, M. y HONORÉ, B. (1999): "Panel Data Models: Some Recent Developments", unpublished manuscript prepared for the Handbook of Econometrics, vol. 5.

BENTOLILA, S. y BERTOLA, G. (1990): "Firing Costs and Labour Demand: How Bad Is Eurosclerosis?", en Review of Economic Studies, vol. 57, $n^{\circ} 3$, pp. 381-402.

BENTOLILA, S. y SAINT-PAUL, G. (1992): "The Macroeconomic Impact of Flexible Labor Contracts: An Application to Spain", en European Economic Review, vol. 36, pp. 1013-1047.

BENTOLILA, S. y SAINT-PAUL, G. (1994): "A Model of Labor Demand with Linear Adjustment Costs”, Labour Economics, vol. 1, pp. 303-326.

BENTOLILA, S. y DOLADO, J. J. (1994): "Labour flexibility and wages: lessons from Spain”, en Economic Policy, pp. 54-99. 
BENTOLILA, S., CAHUC, P., DOLADO, J. y LE BARBANCHON, T. (2010): "Paro y empleo temporal durante la crisis: Una comparación entre Francia y España", en FEDEA. Disponible en: www.crisis09.es/libro_crisis/la_crisis_ de_la_economia_espanola.pdf.

BERTOLA, G. (1990), "Job security, employment and wages", en European Economic review, vol. 34, pp. 851-886.

BERTOLA, G. (1992), "Labor turnover costs and average labor demand", en Journal of Labor Economics, vol.10, n.4, pp. 389-411.

BERTOLA, G.; BOERI, T. y CAZES, S. (1999): "Employment protection and labour market adjustment in OECD countries: Evolving institutions and variable enforcement", en Employment and Training Papers, 48, Organización Internacional del Trabajo (OIT).

BLUNDELL, R. y S. BOND (1998): "Initial Conditions and Moment Restrictions in Dynamic Panel Data Models", en Journal of Econometrics, vol. 87, pp. 115-143.

DOLADO, J. J. y JIMENO, J. F. (2004): "Contratación temporal y costes de despido en España: lecciones para el futuro desde la perspectiva del pasado", en Fundación Alternativas (Documento de Trabajo 48/2004). Disponible en: http://orff.uc3m.es/bitstream/10016/3425/2/DT48-2004-FA.pdf

EECKHOUDT, L. y TREICH, N. (2003): "Adjustment costs, uncertainty, and the level of activity", en Southern Economic Journal, Vol. 69 (4).

GONZÁLEZ, I., PÉREZ, C. y RODRÍGUEZ, J.C. (2012): "Los efectos del incremento del salario mínimo interprofesional en el empleo de los trabajadores inmigrantes en España", en El Trimestre Económico, vol. LXXIX (2), nº 314, abril-junio, pp. 379-414.

GONZÁLEZ MÍNGUEZ, J. y VACAS, C. (2007): "La flexiseguridad como modelo para los mercados de trabajo europeos", en Boletín Económico del Banco de España, noviembre 2007, pp. 71-86.

HAMERMESH, D. (1995): La demanda de trabajo, Madrid: Ministerio de Trabajo y Seguridad Social (colección Economía y Sociología del Trabajo).

HUNT, J. (2000): "Firing Costs, Employment Fluctuations And Average Employment: An Examination of Germany", en Economica, vol. 67, pp. 177202.

LAZEAR, E. P. (1990): “Job Security Provisions and Employment”, en Quarterly Journal of Economics, vol. 105, n 3, pp. 699-726.

LJUNGQVIST, L. (2002): "How do lay-off costs affect employment?", en The Economic Review, no 112, pp. 829-853.

MALO, M. A. (2004): "La evolución institucional del despido disciplinario: una interpretación en términos de un accidente histórico", IX Jornadas de Economía Crítica, Madrid. Disponible en: http://e-archivo.uc3m.es/bitstream/ 10016/3570/1/RHE-2005-XXIII-Malo.pdf

MALO, M. A. (2010): "Costes de despido, temporalidad y reforma laboral en España", documento presentado en el Instituto de Estudios Fiscales. 
MALO, M. A. y TOHARIA, L. (1999): Costes de despido y creación de empleo, Madrid: Ministerio de Trabajo y Asuntos Sociales (colección Estudios).

MALO, M. A. y TOHARIA, L. (2008): "La reforma de los despidos de 2002", en Revista del Ministerio de Trabajo e Inmigración, $\mathrm{n}^{\circ}$ 76, pp. 111-128. Disponible en: http://www.meyss.es/es/publica/pub_electronicas/destacadas/ revista/numeros/76/est05.pdf

NICKELL, S. (1981): "Biases in Dynamic Models with Fixed Effects", en Econometrica, vol. 49, pp. 1417-26.

NICKELL, S. (1991): "Modelos dinámicos de demanda de trabajo", en Ashenfelter y Layard, comps. (1991), Manual de Economía del Trabajo, tomo I, Madrid: Ministerio de Trabajo y Seguridad Social.

OI, W. (1962): "Labor as a quasi-fixed factor", en Journal of Political Economy, $\mathrm{n}^{\circ} 70$, pp. 538-555.

SÁNCHEZ MOLINERO, J. M. (1992): "Relaciones laborales y mercados de trabajo: la experiencia española”, en Cuadernos de Economía, $n^{\circ} 20$, pp. 385414

SIMÓN PÉREZ, H. (2001): "La estructura de salarios pactados en España", en Estudios de Economía Aplicada, vol. 19, nº 003, pp. 171-187. 


\section{Apéndice 1}

\section{REFORMAS LEGISLATIVAS DEL DESPIDO EN ESPAÑA}

\section{Las Reformas de 1994, 1997 y 2002}

A lo largo de las últimas décadas, la legislación laboral española ha sufrido algunos cambios importantes como consecuencia del panorama derivado de la evolución de las relaciones laborales de la década de los ochenta que el Estatuto de los Trabajadores había configurado. Las reformas legislativas de 1994 y 1997 provocaron la inserción de cierta relajación, tratando de flexibilizar las relaciones laborales. El objetivo era canalizar los despidos tratando de evitar un uso estratégico de la legislación que tanto empresas como trabajadores venían realizando. La reducción de las trabas administrativas y burocráticas también ha sido objeto de reforma, y ello ha influido decisivamente en la rebaja del coste total de despido que sufrían las empresas, así como en el periodo de reclamación del trabajador. Asimismo, de la reforma de 1997 surgió un nuevo tipo de contrato "indefinido de fomento del empleo", que rebajaba la indemnización en los casos de improcedencia de un despido económico objetivo por razones económicas, pasando de 45 días a 33 días de salario por año trabajado, con un máximo de 24 mensualidades.

La reforma sobre prestaciones por desempleo de 2002 también ha jugado un papel importante en la configuración del despido en España. En concreto, ha introducido un mecanismo mediante el cual la empresa puede prescindir del pago de los salarios de tramitación reconociendo en un plazo de dos días tras el despido que éste era improcedente y, por ende, poniendo a disposición del trabajador la indemnización correspondiente (Malo y Toharia, 2008). De esta forma, podemos afirmar que la Ley 45/2002 ha supuesto una reducción de los costes de despido de los trabajadores indefinidos, por medio de tres vías: la supresión de los salarios de tramitación, la eliminación de la negociación, y la menor incertidumbre sobre la cuantía de las indemnizaciones, en los casos que se ciñan a esta modalidad de despido. En cambio, también ha supuesto la introducción de un vicio en la forma del despido, e incluso podría suponer un aumento de la cuantía de la indemnización para aquellos despidos que, de otro modo, no fueran a ser declarados improcedentes. Los datos atestiguan la idea de que esta vía de despido se incrementó considerablemente en los años siguientes a la reforma.

\section{La Reforma Laboral de 2010}

En relación con los costes de despido los cambios sustanciales que introduce la Ley 35/2010 podemos resumirlos del siguiente modo:

- Con respecto a los contratos temporales, se introduce una indemnización creciente por despido, que se eleva en un día cada año transcurrido desde 
2010, de forma que en los contratos temporales firmados hasta finales de 2011 se fija en los 8 días por año de servicio actuales, hasta finales de 2012, en 9 días por año de servicio, y así sucesivamente hasta 12 días de salario por año trabajado los que se firmen en el 2015.

- Se reducen las cantidades a abonar por las empresas en despidos económicos, sean individuales o colectivos y excluyendo los disciplinarios, siendo asumidos de forma transitoria por el Fondo de Garantía Salarial (FOGASA) en un $40 \%$.

- Se vuelve a matizar la redacción de las razones económicas, técnicas, organizativas o de producción, admitiéndose ahora en el caso de que existan pérdidas actuales o previstas en la empresa.

- En los contratos de fomento de la contratación indefinida se permite que el empresario aduzca como improcedente el despido por causas objetivas sin necesidad de esperar a la sentencia del Juzgado de lo Social. Así, se permite realizar despidos objetivos que luego serán declarados improcedentes, y tener que abonar únicamente una indemnización de 33 días por año trabajado. Además, al reconocer dicha improcedencia, el empresario también queda exonerado del abono de los salarios de tramitación.

\section{La Reforma Laboral de 2012}

El pasado 10 de febrero se aprobó la Ley 3/2012, de medidas urgentes para la reforma del mercado laboral. El objetivo primordial es favorecer el incremento de la tasa de empleo. Más en concreto, está orientada a las siguientes cuestiones: (1) fomentar la contratación indefinida y creación de empleo; (2) incrementar la flexibilidad interna de las empresas como alternativa al despido, a través de la adaptación de las condiciones laborales a las circunstancias de cada empresa, y (3) reducir la dualidad del mercado de trabajo con medidas centradas sobre todo en la extinción de contratos.

El último punto quizá sea el de un contenido más extenso en la reforma. Interesa especialmente analizar las medidas más importantes que se abordan en él puesto que configura un nuevo marco en la legislación sobre despido de nuestro país. Podemos resumir del siguiente modo los principales cambios en la regulación del despido a través de esta reforma:

- Rebaja los impedimentos para el despido por causas económicas (de 20 días de salario por año trabajado) al estar justificado también cuando la empresa aduzca una disminución persistente de sus ingresos durante al menos tres trimestres.

- Reducción del coste de despido de los contratos indefinidos, de 45 días de salario con un máximo de 48 mensualidades a 33 días de salario con un máximo de 24 mensualidades. 
- Supresión del despido exprés de la Ley 45/2002, que como veremos en el siguiente capítulo es una de las vías más concurridas para despedir desde que se creó.

- Supresión de la autorización administrativa en los despidos colectivos, lo que puede suponer un importante ahorro de tiempo y coste. 


\section{Apéndice 2}

\section{RESOLUCIÓN DEL MODELO}

El modelo se resuelve de forma recursiva.

En el inicio del periodo 2 la empresa conoce tanto $l_{1}$ como la realización del shock $\tilde{\varepsilon}$ y resuelve:

$$
V_{2}\left(l_{1}, \delta, \varepsilon\right)=\max _{l_{2}}\left[B\left(l_{2}, \varepsilon\right)-\delta \cdot g\left(l_{1}-l_{2}\right)\right]
$$

Cuyas condiciones necesarias y suficientes son:

$$
\begin{gathered}
V_{2}^{\prime}\left(l_{1}, \delta, \varepsilon\right)=B^{\prime}\left(l_{2}^{*}, \varepsilon\right)+\delta \cdot g^{\prime}\left(l_{1}^{*}-l_{2}^{*}\right)=0 \\
V_{2}^{\prime \prime}\left(l_{1}, \delta, \varepsilon\right)=B^{\prime \prime}\left(l_{2}^{*}, \varepsilon\right)-\delta \cdot g^{\prime \prime}\left(l_{1}^{*}-l_{2}^{*}\right)<0
\end{gathered}
$$

Tomando en cuenta el ámbito de variación de los valores del shock $(\tilde{\varepsilon})$, es posible hallar el nivel de empleo esperado en el periodo 2:

$$
E\left(l_{2}^{*}\right)=E\left\{l_{2}\left[l_{1}(\delta), \tilde{\varepsilon}, \delta\right]\right\}
$$

Y la ganancia (neta) esperada del periodo 2:

$$
E V\left(l_{1}, \delta, \tilde{\varepsilon}\right)=E\left\{B\left(l_{2}^{*}, \tilde{\varepsilon}\right)-\delta \cdot g\left(l_{1}^{*}-l_{2}^{*}\right)\right\}
$$

El efecto del coste de despido $(d)$ sobre el empleo esperado es:

$$
\frac{d E\left[l_{2}\left(l_{1}(\delta), \tilde{\varepsilon}, \delta\right)\right]}{d \delta}=\frac{\partial E l_{2}^{*}}{\partial \delta}+\frac{\partial E l_{2}^{*}}{\partial l_{1}} \cdot \frac{d l_{1}^{*}}{d \delta}
$$

Los signos de los diferentes términos de la derecha, son:

$$
\begin{gathered}
\frac{\partial E l_{2}^{*}}{\partial \delta}=\frac{-1}{E V_{2}^{\prime \prime}} \cdot \frac{\partial E V_{2}^{\prime}}{\partial \delta}=\frac{-1}{E V_{2}^{\prime \prime}} \cdot E\left[g^{\prime}\left(l_{1}^{*}-l_{2}^{*}\right)\right]>0 ; \text { si } l_{2}^{*}<l_{1}^{*} \\
\frac{\partial E l_{2}^{*}}{\partial l_{1}^{*}}=\frac{-1}{E V_{2}^{\prime \prime}} \cdot \frac{\partial E V_{2}^{\prime}}{\partial l_{1}^{*}}=\frac{-\delta}{E V_{2}^{\prime \prime}} \cdot E\left[g^{\prime \prime}\left(l_{1}^{*}-l_{2}^{*}\right)\right]>0 ; \text { si } l_{2}^{*}<l_{1}^{*} \text { y } g^{\prime \prime}>0
\end{gathered}
$$

Para obtener el valor de $\frac{d l_{1}^{*}}{d \delta}$ resolvemos el problema del periodo 1. Para ello incorporamos los resultados esperados del periodo 2 :

$$
V_{1}(\delta, \tilde{\varepsilon})=\max _{l_{1}}\{B\left(l_{1}\right)+\underbrace{E\left\{B\left(l_{2}^{*}, \tilde{\varepsilon}\right)-\delta \cdot g\left(l_{1}^{*}-l_{2}^{*}\right)\right\}}_{E V\left(l_{1}, \delta, \tilde{\varepsilon}\right)}\}
$$


Cuyas condiciones necesarias y suficientes implican:

$$
\begin{gathered}
V_{1}^{\prime}(\delta, \tilde{\varepsilon})=B^{\prime}\left(l_{1}^{*}\right)-\delta \cdot E\left\{g^{\prime}\left(l_{1}^{*}-l_{2}^{*}\right)\right\}=0 \\
V_{1}^{\prime \prime}(\delta, \tilde{\varepsilon})=B^{\prime \prime}\left(l_{1}^{*}\right)-\delta \cdot E\left\{g^{\prime \prime}\left(l_{1}^{*}-l_{2}^{*}\right)\right\}<0
\end{gathered}
$$

De donde obtenemos la demanda de input presente: $l_{1}^{*}=l_{1}(\delta)$

Podemos calcular el signo del efecto del coste de despido sobre dicho nivel de empleo:

$$
\frac{\partial l_{1}^{*}}{\partial \delta}=\frac{-1}{V_{1}^{\prime \prime}} \cdot \frac{\partial V_{1}^{\prime}}{\partial \delta}=\frac{1}{V_{1}^{\prime \prime}} \cdot E\left[g^{\prime}\left(l_{1}^{*}-l_{2}^{*}\right)\right]<0 ; \text { si } l_{2}^{*}<l_{1}^{*}
$$

